\title{
Correction to: Physical exercise augmented cognitive behaviour therapy for older adults with generalised anxiety disorder (PEXACOG): study protocol for a randomized controlled trial
}

\author{
Silje Haukenes Stavestrand ${ }^{1,2^{*}}$, Kristine Sirevåg ${ }^{1,2}$, Inger Hilde Nordhus ${ }^{1,3}$, Trond Sjøb $\varnothing^{2}$, Trygve Bruun Endal ${ }^{2}$, \\ Hans M. Nordahl ${ }^{4,9}$, Karsten Specht ${ }^{1}$, Åsa Hammar ${ }^{1}$, Anne Halmøy ${ }^{5,11}$, Egil W. Martinsen ${ }^{3,10}$, Eva Andersson ${ }^{6}$, \\ Helene Hjelmervik', Jan Mohlman ${ }^{7}$, Julian F. Thayer ${ }^{8}$ and Anders Hovland ${ }^{1,2}$
}

Correction to: Trials (2019) 20:174

https://doi.org/10.1186/s13063-019-3268-9

Following the publication of our article [1], we have become aware of one error in the exclusion criteria, inconsistencies in Figs. 1 and 2, and a typo in the reference list.

Exclusion criterion \# 10 is incorrect.

Correction:

(10) participating regularly in $60 \mathrm{~min}$ of moderate intensity physical exercise per week, divided across 2 or more bouts of physical exercise.

The physical exercise exclusion criteria are omitted from Fig. 1 in the original publication. The correct version of Fig. 1 is included here.

In Fig. 2, the interim testing contains three tests which are not conducted at this point of measure. These are The Five-Minute Pyramid test, The Biering-Sørensen Test, and the Dumbbell Arm Press Test. The correct version of Fig. 2 is included here.

We have discovered a typo in the reference for the Expectancy / Credibility Scale. The correct reference is:

\footnotetext{
The original article can be found online at https://doi.org/10.1186/s13063019-3268-9

* Correspondence: silje.h.stavestrand@uib.no

${ }^{1}$ Faculty of Psychology, University of Bergen, Box 7800, NO-5020 Bergen, Norway

${ }^{2}$ Solli DPS, Osvegen 15, NO-5228 Nesttun, Norway

Full list of author information is available at the end of the article
}

Borkovec, T. D. and S. D. Nau (1972). "Credibility of analogue therapy rationales." Journal of Behavior Therapy and Experimental Psychiatry 3(4): 257-260.

The original article can be found online at https://doi.org/10.1186/s13063019-3268-9.

\section{Author details \\ ${ }^{1}$ Faculty of Psychology, University of Bergen, Box 7800, NO-5020 Bergen, Norway. ${ }^{2}$ Solli DPS, Osvegen 15, NO-5228 Nesttun, Norway. ${ }^{3}$ Faculty of Medicine, University of Oslo, Box 1078, Blindern, NO-0316 Oslo, Norway. ${ }^{4}$ Department of Mental Health, Norwegian University of Science and Technology, Box 8905, NO-7491 Trondheim, Norway. ${ }^{5}$ Faculty of Medicine, K.G. Jebsen Centre for Neuropsychiatric Disorders, University of Bergen, Box 7800, NO-5020 Bergen, Norway. ${ }^{6}$ The Swedish School of Sport and Health Sciences, GIH, Box 5626, SE-114 86 Stockholm, Sweden. 7 Department of Psychology, William Paterson University, 300 Pompton Road, Wayne, NJ 07470, USA. ${ }^{8}$ Department of Psychology, Ohio State University, 1835 Neil Avenue, Columbus, OH 43210, USA. ' St.Olavs Hospital HF, Nidaros DPS, Box 3250, Sluppen, NO-7006 Trondheim, Norway. ${ }^{10}$ Division of Mental Health and Addiction, Oslo University Hospital, Oslo, Norway. ${ }^{11}$ Kronstad DPS/Division of Psychiatry, Haukeland University Hospital, Box 1400, NO-5021 Bergen, Norway.}

Published online: 06 April 2020

\section{Reference}

1. Stavestrand $\mathrm{SH}$, et al. Physical exercise augmented cognitive behaviour therapy for older adults with generalised anxiety disorder (PEXACOG): study protocol for a randomized controlled trial. Trials. 2019;20:174. https://doi. org/10.1186/s13063-019-3268-9.

(c) The Author(s). 2020 Open Access This article is licensed under a Creative Commons Attribution 4.0 International License, which permits use, sharing, adaptation, distribution and reproduction in any medium or format, as long as you give appropriate credit to the original author(s) and the source, provide a link to the Creative Commons licence, and indicate if changes were made. The images or other third party material in this article are included in the article's Creative Commons licence, unless indicated otherwise in a credit line to the material. If material is not included in the article's Creative Commons licence and your intended use is not permitted by statutory regulation or exceeds the permitted use, you will need to obtain permission directly from the copyright holder. To view a copy of this licence, visit http://creativecommons.org/licenses/by/4.0/ The Creative Commons Public Domain Dedication waiver (http://creativecommons.org/publicdomain/zero/1.0/) applies to the data made available in this article, unless otherwise stated in a credit line to the data. 


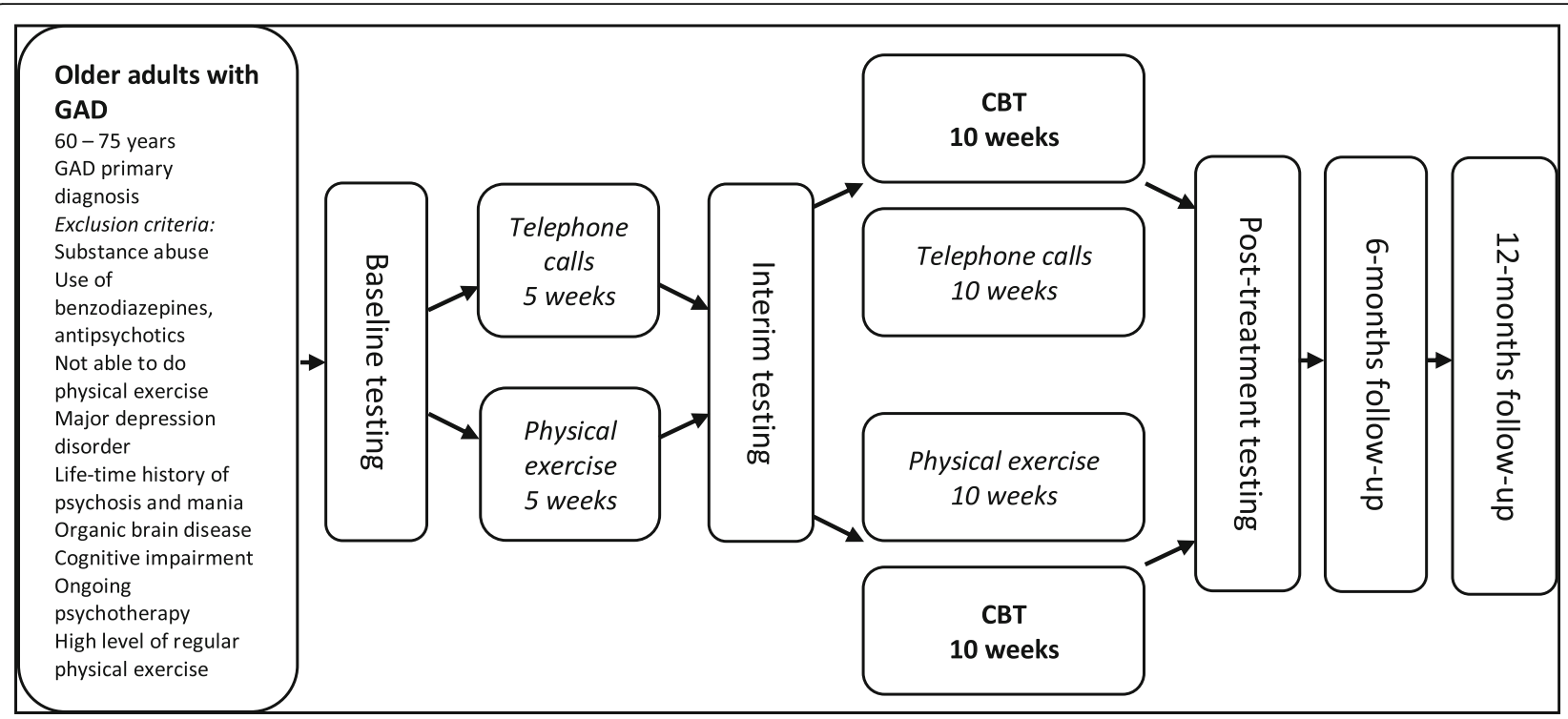

Fig. 1 Study design. Visual presentation of the study design, including sample, assessments, and interventions. GAD, generalised anxiety disorder; CBT, cognitive behaviour therapy 


\begin{tabular}{|c|c|c|c|c|c|c|c|c|c|}
\hline \multirow[b]{3}{*}{ TIMEPOINT } & \multicolumn{9}{|c|}{ STUDY PERIOD } \\
\hline & \multicolumn{3}{|c|}{ Pre-treatment } & \multicolumn{3}{|l|}{ Treatment } & \multicolumn{3}{|c|}{ Post-treatment } \\
\hline & $W-x$ & W -1 & 0 & W 1-5 & W6 & W 7-16 & W 17 & M 10 & M 16 \\
\hline & $\begin{array}{l}\text { Enrol } \\
\text { ment }\end{array}$ & Baseline & $\begin{array}{l}\text { Alloc } \\
\text { ation }\end{array}$ & $\begin{array}{l}\text { Initial phase: } \\
\text { PE or AP }\end{array}$ & $\begin{array}{l}\text { Interrim } \\
\text { testing }\end{array}$ & $\begin{array}{l}\text { CBT + } \\
\text { PE or AP }\end{array}$ & $\begin{array}{l}\text { Post } \\
\text { testing }\end{array}$ & $\begin{array}{l}\text { 6-m F- } \\
U\end{array}$ & $\begin{array}{l}12-m \\
F-U\end{array}$ \\
\hline \multicolumn{10}{|l|}{ ENROLMENT: } \\
\hline Eligibility screen & $\mathrm{X}$ & & & & & & & & \\
\hline Informed consent & $\mathrm{X}$ & & & & & & & & \\
\hline Allocation & & & $\mathrm{X}$ & & & & & & \\
\hline \multicolumn{10}{|l|}{ INTERVENTIONS: } \\
\hline \multicolumn{10}{|l|}{ CBT + Physical Exercise (PE) } \\
\hline \multicolumn{10}{|l|}{ CBT + Attention Placebo (AP) } \\
\hline \multicolumn{10}{|l|}{ ASSESSMENTS: } \\
\hline \multicolumn{10}{|l|}{ Interviews } \\
\hline ADIS-IV (primary outcome measure) & $x$ & & & & & & $x$ & & \\
\hline M.I.N.I. Interview & $x$ & & & & & & & & \\
\hline Mini Mental State Examination & $\mathrm{X}$ & & & & & & & & \\
\hline Demography & $\mathrm{X}$ & & & & & & & & \\
\hline \multicolumn{10}{|l|}{ Questionnaires } \\
\hline GAD-7 & $\mathrm{X}$ & $\mathrm{X}$ & & $\mathrm{X}$ & $\mathrm{X}$ & $\mathrm{X}$ & $\mathrm{X}$ & $\mathrm{X}$ & $\mathrm{X}$ \\
\hline Beck Anxiety Inventory & & $\mathrm{X}$ & & & $\mathrm{X}$ & & $\mathrm{X}$ & $\mathrm{X}$ & $\mathrm{X}$ \\
\hline PSWQ (primary outcome measure) & & $\mathrm{X}$ & & & $\mathrm{X}$ & & $\mathrm{X}$ & $\mathrm{X}$ & $\mathrm{X}$ \\
\hline Geriatric Anxiety Inventory & & $x$ & & & $X$ & & $x$ & $x$ & $\mathrm{X}$ \\
\hline Beck Depression Inventory - II & & $\mathrm{X}$ & & & $\mathrm{X}$ & & $x$ & $\mathrm{X}$ & $x$ \\
\hline Quality of life inventory & & $\mathrm{X}$ & & & $\mathrm{X}$ & & $\mathrm{X}$ & $\mathrm{X}$ & $\mathrm{X}$ \\
\hline Bergen Insomnia Scale & & $\mathrm{X}$ & & & $x$ & & $\mathrm{X}$ & $x$ & $\mathrm{X}$ \\
\hline Ecpectancy / Credibility scale & & $\mathrm{X}$ & & & & & $\mathrm{x}$ & & $\mathrm{X}$ \\
\hline IPAQ & & $\mathrm{X}$ & & & $\mathrm{X}$ & & $\mathrm{X}$ & & $\mathrm{X}$ \\
\hline \multicolumn{10}{|l|}{ Objective measures } \\
\hline Actigraph - 7 days & & $\mathrm{X}$ & & & & & $\mathrm{X}$ & & $\mathrm{X}$ \\
\hline Cortisol - 3 saliva samplers & & $\mathrm{X}$ & & & $x$ & & $\mathrm{X}$ & $x$ & $\mathrm{X}$ \\
\hline DNA - saliva sample & & $\mathrm{X}$ & & & & & & & \\
\hline HRV & & $\mathrm{X}$ & & & $\mathrm{X}$ & & $\mathrm{X}$ & $\mathrm{X}$ & $\mathrm{X}$ \\
\hline BDNF - blood sample & & $X X$ & & & $X X$ & & $X X$ & $X X$ & $X X$ \\
\hline Ekblom-Bak Cycle test & & $\mathrm{X}$ & & & $\mathrm{X}$ & & $x$ & $\mathrm{X}$ & $\mathrm{X}$ \\
\hline Five-Minute Pyramid Test & & $\mathrm{X}$ & & & & & $\mathrm{X}$ & $\mathrm{X}$ & $\mathrm{X}$ \\
\hline Biering-Sørensen test & & $\mathrm{X}$ & & & & & $\mathrm{X}$ & $\mathrm{X}$ & $\mathrm{X}$ \\
\hline Timed sit-to-stand test & & $X$ & & & $\mathrm{X}$ & & $\mathrm{X}$ & $x$ & $x$ \\
\hline $45^{\circ}$ sit-up test & & $\mathrm{X}$ & & & $x$ & & $\mathrm{X}$ & $\mathrm{X}$ & $\mathrm{X}$ \\
\hline Dumbbell arm press test & & $\mathrm{X}$ & & & & & $\mathrm{X}$ & $\mathrm{X}$ & $\mathrm{X}$ \\
\hline \multicolumn{10}{|l|}{ Neuropsychological testing } \\
\hline Vocabulary (WASI) & & $x$ & & & & & $\mathrm{X}$ & & $\mathrm{X}$ \\
\hline Matrix Reasoning (WASI) & & $\mathrm{X}$ & & & & & $\mathrm{X}$ & & $\mathrm{X}$ \\
\hline Grooved Pegboard Test & & $X$ & & & & & $x$ & & $x$ \\
\hline Coding (WAIS-IV) & & $\mathrm{X}$ & & & & & $\mathrm{X}$ & & $\mathrm{X}$ \\
\hline Numbers (WAIS-IV) & & $\mathrm{X}$ & & & & & $\mathrm{X}$ & & $\mathrm{X}$ \\
\hline Wisconsin Card Sorting Test & & $\mathrm{X}$ & & & & & $x$ & & $\mathrm{X}$ \\
\hline D-KEFS (TMT, VF, CWIT) & & $\mathrm{X}$ & & & & & $\mathrm{X}$ & & $\mathrm{X}$ \\
\hline California Verbal Learning Test & & $\mathrm{X}$ & & & & & $\mathrm{X}$ & & $\mathrm{X}$ \\
\hline \multicolumn{10}{|l|}{ Subsample of 40 participants } \\
\hline Magnetic resonnance imaging & & $\mathrm{X}$ & & & & & $\mathrm{X}$ & & $\mathrm{X}$ \\
\hline \multicolumn{10}{|c|}{ Fig. 2: SPIRIT Figure. Schedule of enrolment, interventions and assessments. } \\
\hline \multicolumn{10}{|c|}{$\begin{array}{l}\text { Abbreviations: W Week; M Month; F-U Follow- } \\
\text { up }\end{array}$} \\
\hline
\end{tabular}

Fig. 2 (See legend on next page.) 
(See figure on previous page.)

Fig. 2 Standard protocol items: recommendation for interventional trials (SPIRIT) figure. Schedule of enrolment, interventions and assessments. Overview of the measures applied in the study. CBT, cognitive behaviour therapy; ADIS-IV, Anxiety Disorders Interview Schedule for DSM-IV; M.I.N.I., Mini International Neuropsychiatric Interview; PSWQ, Penn State Worry Questionnaire; GAD-7, Generalized Anxiety Disorder 7-item scale; IPAQ, International Physical Activity Questionnaire; HRV, heart rate variability; BDNF, brain-derived neurotropic factor; WASI, Wechsler Abbreviated Scale of Intelligence; WAIS-IV, Wechsler Adult Intelligence Scale - Fourth Edition; D-KEFS, Delis-Kaplan Executive Function System; TMT, TrailMaking Test; VF, Verbal Fluency; CWIT, Color Word Interference Test 